\title{
Objetos digitales de aprendizaje en entornos virtuales para fomentar la escritura: percepciones de los estudiantes
}

\section{Digital learning objects in virtual environments to encourage writing: students' perceptions}

\author{
FLORES-GONZÁLEZ, Norma †*, FLORES-GONZÁLEZ, Efigenia, CASTELÁN-FLORES, Vianey y \\ ZAMORA-HERNANDEZ, Mónica
}

Benemérita Universidad Autónoma de Puebla, Facultad de Lenguas, México.

Benemérita Universidad Autónoma de Puebla, Preparatoria Regional Enrique Cabrera Barroso, México.

ID $1^{\text {er }}$ Autor: Norma, Flores-González / ORC ID: 0000-0002-4967-8854, Researcher ID Thomson: S-6917-2018, CVU CONACYT ID: 957036

ID $1^{\text {er }}$ Coautor: Efigenia, Flores-González / ORC ID: 0000-0002-8340-9340, Researcher ID Thomson: S-5923-2018, CVU CONACYT ID: 333959

ID $2^{\text {do }}$ Coautor: Vianey, Castelán-Flores / ORC ID: 0000-0001-8687-2552, Researcher ID Publons: 3071615

ID $3^{\text {er }}$ Coautor: Mónica, Zamora-Hernández / ORC ID: 0000-0002-7012-4805, Researcher ID Publons: 3071599

DOI: $10.35429 / J I T .2020 .24 .7 .1 .12$

Recibido: Julio 10, 2020; Aceptado Diciembre 30, 2020

\begin{abstract}
Resumen
Las Tecnologías de la información y la comunicación tienen un lugar fundamental en el campo de la educación al ofrecer a los estudiantes una manera atractiva para la apropiación del conocimiento de una lengua extrajera. Un ejemplo de ello, son los objetos digitales de aprendizaje (ODA) que proveen aprendizajes con características distintas a la clase tradicional. Este artículo tiene por objetivo conocer las percepciones de los alumnos de nivel universitario con respecto al uso de ODAs en un ambiente virtual para promover la competencia escrita en inglés como lengua extranjera. Con el fin de caracterizar dichas percepciones se recurrió al enfoque cuantitativo con alcance descriptivo y un cuestionario tipo likert con cuatro categorías de respuesta para recolectar datos y conocer sus opiniones. Como resultado se obtuvo la descripción precisa de los beneficios al utilizar el modelo instruccional tales como: aprendizaje activo, redacción efectiva y autónoma mediante estrategias de redacción y herramientas digitales para la corrección y edición, registro de cambios positivos en el desempeño de los estudiantes con tintes motivacionales e incursión en la redacción de géneros distintos. Finalmente, el estudio aporta una contribución teórica con respecto al uso de herramientas digitales en plataforma Moodle para desarrollar la competencia escrita.
\end{abstract}

Competencia escrita, Objetos digitales de aprendizaje, Entornos virtuales

\begin{abstract}
Information and communication technologies have an elemental place in the field of education by offering students an attractive way for the appropriation of knowledge of a foreign language. An example of this is the digital learning objects (LOs) that provide learning with characteristics different from the traditional class. This article aims to know the perceptions of university-level students regarding the use of LOs in a virtual environment to promote written competence in English as a foreign language. A quantitative descriptive approach took place to characterize these perceptions, as well as a Likert-type questionnaire with four response categories to collect data and identify students' opinions. As a result, we obtain an accurate description of the benefits of using the instructional model such as active learning, autonomous writing through writing strategies and digital tools for correction and editing, identification of positive changes in the students' performance with motivational features, and incorporation of writing in different genres. Finally, the study provides a theoretical contribution regarding the use of digital tools on the Moodle platform to develop written competence.
\end{abstract}

Written competence, Digital learning objects, Virtual environments

Citación: FLORES-GONZÁLEZ, Norma, FLORES-GONZÁLEZ, Efigenia, CASTELÁN-FLORES, Vianey y ZAMORAHERNANDEZ, Mónica. Objetos digitales de aprendizaje en entornos virtuales para fomentar la escritura: percepciones de los estudiantes. Revista de Tecnologías de la Información. 2020. 7-24:1-12.

\footnotetext{
* Correspondencia del Autor (Email: norma-fg@hotmail.com)

$\dagger$ Investigador contribuyendo como primer autor.
} 


\section{Introducción}

La escritura es considerada como el medio para socializar información, validar, y construir conocimiento. En este punto, el idioma inglés representa un elemento fundamental, ya que la mayoría de la información especializada se encuentra en este idioma, por tanto, el escribir y leer en dicho idioma representa una puerta de acceso a fuentes de datos actualizadas. Al respecto, Berrios (2004) puntualiza que la tecnología permite no solo la comunicación sino también el desenvolvimiento de nuevas habilidades y con ello, formas de construir el conocimiento.

Por otro lado, la incorporación exitosa de un alumno universitario a su comunidad radica en el desarrollo de habilidades lingüísticas y discursivas alineadas a su campo académico en términos de la producción y comprensión de textos.

Existen varias concepciones de escritura que coinciden en describirla como un proceso. Salvador $(2000$, p.18) puntualiza que "el control mental de estas decisiones se llama proceso metacognitivo". Para Canale y Swain (1980), es necesario dominar cuatro elementos básicos de la competencia comunicativa para lograr una competencia escrita aceptable a saber: competencia gramatical, estratégica, discursiva y sociolingüística. No obstante, cualquiera que sea la definición y el enfoque, estrategia o modelo para propiciar y enseñar a escribir, resulta ser un quehacer demandante y complicado de llevar a cabo y más aún si es en una segunda lengua.

Esta competencia es un proceso que requiere desarrollo de habilidades específicas tales como planificación, redacción y revisión, así como de estrategias cognitivas, metacognitivas y de autorregulación para dar coherencia y cohesión al texto.

Afortunadamente, con la incursión de la tecnología en el proceso educativo, se identifican varias aplicaciones y herramientas digitales que fomentan su desarrollo de manera atractiva, activa y autónoma en un ambiente colaborativo y amigable. Un ejemplo claro lo constituyen los objetos digitales de aprendizaje, los cuales permiten un aprendizaje colaborativo y motivador con pensamiento crítico y reflexivo.
De hecho, son detonadores de habilidades interpersonales y de relaciones sociales entre estudiantes dando sentido de pertenencia a una comunidad virtual.

Tomando en cuenta estos antecedentes, la presente investigación tiene por objetivo conocer cuáles son las percepciones de los alumnos cuando trabajan en entornos virtuales con materiales digitales como los objetos digitales de aprendizaje para desarrollar su proceso de escritura en inglés como lengua extranjera.

\section{Marco Teórico}

\section{Escritura}

Actualmente, la escritura ha cobrado relevancia en la adquisición de la lengua inglesa. No obstante, dicha actividad se concibe como compleja, incluso en el nivel superior debido a los elementos contextuales y cognitivos que demanda (Prior, 2006).

Peyton et al., (1994) mencionan que cuando el estudiante elabora textos en una lengua extranjera, experimenta obstáculos como aquellos propios que solicita el proceso de escritura y el de la lengua extranjera, la cual está conociendo y se ve limitado con respecto al bagaje léxico, sistematización de palabras en oraciones, párrafos y en general la estructura del texto. Por tanto, los alumnos cuyo nivel de proficiencia en L2 es básico, usualmente desisten a escribir, al considerar dicha acción como una tarea demandante y complicada de realizar. Esto sucede porque el proceso se ve afectado por interrupciones recurrentes entre el diccionario y la traducción de la L1 a la L2.

Es en esta etapa, cuando el quehacer docente recobra importancia al brindarles modelos útiles de acercamiento y comprensión a dicho proceso, ya sea de manera individual o colaborativa.

Hasta el momento, se ha dicho que la escritura es un proceso. Sin embargo, es necesario puntualizar que dicho proceso no es lineal ni definitivo, ya que su objetivo es la transmisión y socialización de la información y el conocimiento donde las ideas se formulan, revisan, editan, corrigen y reeditan con la finalidad de plasmar exactamente lo que se planea decir.

FLORES-GONZÁLEZ, Norma, FLORES-GONZÁLEZ, Efigenia, CASTELÁN-FLORES, Vianey y ZAMORAHERNANDEZ, Mónica. Objetos digitales de aprendizaje en entornos virtuales para fomentar la escritura: percepciones de los estudiantes. Revista de Tecnologías de la Información. 2020 
Cabe indicar que la revisión es una fase colaborativa fundamental del proceso de escritura entre alumno y docente, donde el apoyo del último actor se realiza en dos planos paralelamente. En el primero, revisa y realimenta lo que el escritor (estudiante) ha expresado. En el segundo, identifica e indica errores gramaticales, ortográficos, así como elementos de cohesión y coherencia.

Para ello, los entornos virtuales y especialmente los ODA promueven esa colaboración para revisar dichos textos y dar puntos de vista o realimentación proactiva a un escrito en un ambiente virtual amigable que puede ser sincrónico o asincrónico.

\section{Procesos de la escritura}

En el desarrollo de la competencia escrita se identifican varios procesos dando prioridad a la movilización de saberes procedimentales tales como (González, Rodríguez \& Ledo, 2019):

- $\quad$ Identificar el contenido temático.

- $\quad$ Elegir el propósito del escrito y el registro. Es decir, definir si el objetivo es informar, explicar, argumentar, describir, etc.

- Organizar el escrito. Esto se realiza mediante la formulación de posibles preguntas que el lector elabora.

- $\quad$ Planificar, ordenar y sistematizar las ideas.

- $\quad$ Integrar la información en párrafos.

- Usar estrategias ad hoc al propósito del texto el cual dependerá del autor.

- Emplear signos de puntuación adecuados.

- Aplicar estrategias metacognitivas durante el proceso de redacción, edición, revisión y corrección.

Por otro lado, también hay procesos cognitivos que fomentan la escritura vista como una actividad comunicativa, compuesta por elementos lingüísticos, mentales y estilísticos.
De acuerdo con Cassany (2005, p. 8), "las habilidades, microhabilidades lingüísticas y cognitivas que son adquiridas y desarrolladas por el individuo son primordiales en el proceso completo de composición de un texto".

A continuación, se enlistan dichos procesos cognitivos:

De planeación. Permite al estudiante preparase con respecto al tema que va a escribir a través de la búsqueda de información, para su posterior selección y organización. En esta fase, el escritor identifica el receptor y el propósito del escrito (García, 2007).

De redacción. Una vez que el escritor tiene la información suficiente, deberá plasmar sus ideas de manera escrita recurriendo a componentes sintácticos, lingüísticos, pragmáticos y semánticos mediante un registro formal y acorde a su audiencia. Esta fase implica la elaboración de borradores, los cuales evidenciaran infinidad de cambios hasta que se obtenga la versión final, que se da cuando el texto expresa lo que el escritor quiere decir (Serrano y Peña, 2003).

De revisión. Consiste en analizar el escrito para identificar errores $\mathrm{y}$, en general, examinar la coherencia y, por tanto, la cohesión del texto, con el propósito de obtener una versión adecuada para su publicación.

Finalmente, es importante destacar que dichas fases no son lineales, por el contrario, interactúan entre sí durante el complejo proceso de escritura.

\section{Medios digitales para desarrollar la competencia escrita}

En el estado del arte se identifican herramientas digitales, objetos digitales de aprendizaje, software y aplicaciones para robustecer la competencia escrita, particularmente en inglés como Lengua Extranjera.

Las herramientas digitales de trabajo colaborativo y ambientes virtuales forman parte de la variedad de elementos tecno-pedagógicos que facilitan la actividad de alumnos y maestros, especialmente en estos tiempos de confinamiento y contingencia. 
De hecho, los procesos de enseñanza y de aprendizaje de lenguas $\mathrm{y}$, específicamente, los procesos de escritura se ven notoriamente afectados por las aplicaciones, herramientas digitales y uso de la web 2.0 y 3.0. Un ejemplo lo constituyen los ODA vistos como tecnología instruccional que fomentan el aprendizaje colaborativo y autónomo.

Esta tecnología está basada en una metodología digital enfocada en objetos, el cual se refiere a crear componentes 0 sistemas que puedan ser reutilizables en otros programas $\mathrm{y}$ adaptados a distintas tareas $\mathrm{y}$ actividades (Willey, 2000).

Al respecto, López, Romero y Ramírez (2008), afirman que los ODA favorecen el dominio de los contenidos programáticos, lo que facilita la habilidad y fluidez de la escritura de una segunda lengua.

Otra de sus bondades es que pueden integrarse en recursos educativos abiertos (REA), cuya característica radica en que son materiales de alta calidad, de dominio público, gratuito y de fácil acceso para que los estudiantes lo utilicen en el momento que lo consideren pertinente (Burgos, 2009). Sin embargo, para la enseñanza y aprendizaje de la escritura en un segundo idioma, un ODA debe poseer características elementales como experiencias reales con un significado que motive y cubra los requerimientos del estudiante con respecto al idioma. De esta manera, los ODA son eficientes si cumplen con dichas características (Barritt, Chuck \& Alderman, 2004).

Distintas investigaciones señalan que el uso de herramientas digitales también promueve la competencia escrita.

Kessler, (2009), Chao y Lo (2009) y González y García-Romeu (2010) afirman que el desarrollo de la escritura colaborativa centrada en la redacción de un mismo texto se logra a partir del uso de wikis.

Aunado a lo anterior, Ward (2004), Wu (2005) y Zhang (2009) consideran que los blogs también favorecen la fluidez de la escritura e interacción entre docente- alumno y alumnoalumno.
Por su parte, Flores-González, et al., (2018) afirman que los organizadores gráficos mediados por tecnología promueven el análisis y síntesis de la información lo que favorece la comprensión, comunicación y redacción de un tema en específico.

\section{Entornos virtuales para fomentar la escritura}

Para Quiroz (2011), un entorno virtual es un escenario tratado pedagógicamente para enseñar y generar aprendizajes a través de una plataforma, tareas y recursos materiales.

Estos espacios brindan las condiciones idóneas para que los sujetos interactúen, generen nuevos conocimientos, procesos de síntesis y reflexión. Los entornos virtuales son un espacio de oportunidades para ampliar el abanico de herramientas que contribuyen a la calidad del aprendizaje de la escritura, cuyo sustento radica en un aprendizaje cognitivo. De acuerdo con Beltrán (2003)

Los ordenadores pueden apoyar el
pensamiento reflexivo de los estudiantes
porque les permiten aprender planificando las
actividades, controlando sus resultados,
evocando lo que ya saben, creando
conocimientos nuevos, modificando los
viejos, aprendiendo de los errores,
consolidando los aciertos, en suma, tomando
decisiones respecto a la cadena de la
construcción del conocimiento (p.13).

Es así como la implementación de un entorno virtual posiciona a los sujetos en diferentes contextos en los que es indispensable escribir, incluso desde la comunicación entre los mismos estudiantes y entre el estudiantedocente, identificando un receptor real para obtener una realimentación sobre su escritura (Enríquez, 2011). Usualmente, esta interacción se promueve en un foro virtual que genera un trabajo colaborativo rico en la construcción de significados (Garrison y Anderson, 2005; Gisbert, Cabero y Llorente, 2007).

Es menester mencionar que en la escritura colaborativa se identifica una inmensa variedad de aplicaciones para sistemas de aprendizaje a distancia (e-learning), que integran herramientas de revisión y coedición textual innovadoras y atractivas para los estudiantes, tales como Blackboard o Moodle entre otras. 
A pesar de que estas plataformas no integran funciones de edición y revisión colaborativa para las tareas de la competencia escrita en alumnos universitarios, y con propósitos específicos, se pueden complementar con herramientas digitales externas o software como son Grammarly, Spell Check Plus, Reverso, Language Tool, Whitesmoke Writer, Scribens y Turnitin, entre otras.

A partir de lo anterior se afirma que el proceso de enseñanza aprendizaje del idioma inglés, especialmente el de la escritura se ve beneficiado por las herramientas de la información y la comunicación.

En el escenario educativo, dichas herramientas favorecen en el estudiante un pensamiento socio cognitivo para ingresar, deconstruir, producir y socializar el conocimiento, características que demanda el proceso de escritura del inglés (Torres, Ayala \& Vinasco, 2010).

Por otra parte, los hallazgos de la investigación de Haan y van Esch (2005) demuestran que la práctica de la lengua extranjera a través de un entorno virtual provee a los estudiantes de fluidez en la lengua, aspecto determinante para que el sujeto escriba oraciones compuestas y complejas para producir textos extensos.

Otros estudios de escritura académica en entornos virtuales realizados por Greene (2000) e Hirvela (2005) revelan que la escritura electrónica es determinante para la evaluación del conocimiento y el desempeño de los sujetos, haciendo énfasis en la importancia de trabajar textos de opinión que demanden citación y pensamiento crítico para el proceso de composición.

Por último. los ambientes virtuales en línea, permiten romper las barreras físicas, y crear espacios de interacción activa, participativa y eficiente para la educación (Paquienséguy \& Pérez, 2010).

\section{Metodología}

Con el afán de analizar este fenómeno de estudio, se recurrió a la metodología cuantitativa con un diseño transversal y alcance descriptivo. Por lo que respecta al instrumento, se utilizó un cuestionario de escala tipo Likert para conocer la percepción de los sujetos con respecto al trabajo en entornos virtuales con materiales digitales como los ODA para desarrollar su proceso de escritura.

\section{Muestra}

Estuvo compuesta por 20 sujetos que comparten las siguientes características:

- Dificultades para escribir en inglés

- $\quad$ Nivel bajo de proficiencia en L2

- Clases de redacción académica 4 horas a la semana y 4 horas en plataforma utilizando ODAs (con uso de herramientas digitales para la planeación, redacción, revisión y edición de textos escritos) como medio para desarrollar la competencia escrita.

El estudio se realizó en un curso de Redacción Académica de la Licenciatura en Enseñanza del Inglés de la Benemérita Universidad Autónoma de Puebla durante el otoño de 2019.

\section{Resultados}

A partir de los datos recabados, se obtuvieron los siguientes resultados:

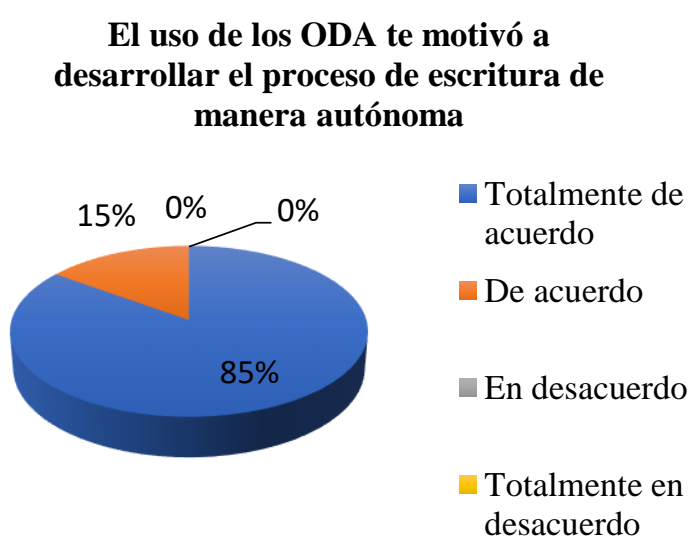

Gráfico 1 El uso de los ODA te motivó a desarrollar el proceso de escritura de manera autónoma 
Como se observa en el gráfico, el 100\% de la muestra está de acuerdo en que los ODA permiten desarrollar la competencia escrita de manera autónoma. Esto confirma los hallazgos de Sharma y Barrett (2007) quienes sostienen que uno de los beneficios de trabajar en entornos virtuales es que el estudiante es dueño de su tiempo, conveniencia y ritmo. Estos elementos aportan significativamente al proceso de aprendizaje, sobre todo cuando se tiene que satisfacer distintos estilos de aprendizaje y niveles de proficiencia por parte de los alumnos.

\section{El uso del ODA promueve un} aprendizaje activo

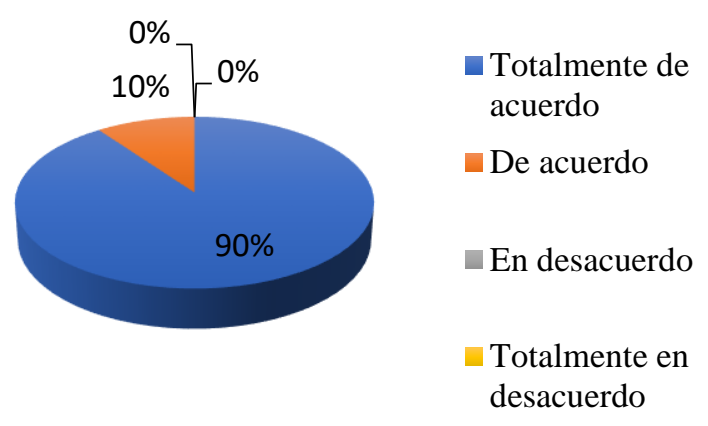

Gráfico 2 El uso del ODA promueve un aprendizaje activo

El $100 \%$ de la muestra manifiesta estar de acuerdo con el desarrollo del aprendizaje activo mediante el uso de este modelo instruccional consistente en ODAs. Estos resultados demuestran lo señalado por Paquienséguy \& Pérez (2010), con respecto a las bondades de los ambientes virtuales para crear interacciones activas.

Como se sabe, el aprendizaje activo se da cuando los estudiantes están construyendo activamente ideas y habilidades a partir de patrones, y en este caso, los ODA proporcionan dichos modelos a través de los cuales se propicia la escritura.

Por otro lado, las afirmaciones de la muestra implican que, al construir activamente el conocimiento, están modificando y transformando (Bazerman et al., 2005) su proceso de aprendizaje en la lengua extranjera, ventaja que aporta únicamente el desarrollo de la escritura integradora donde convergen patrones culturales, históricos, y lingüísticos.

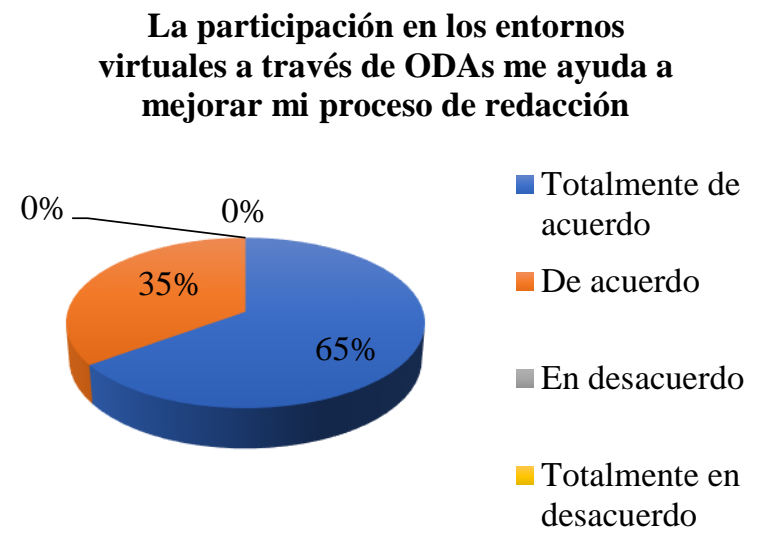

Gráfico 3 La participación en los entornos virtuales me ayuda a mejorar mi proceso de redacción

Debido a la naturaleza de la modalidad donde los estudiantes tienen tareas entre clases presenciales, la participación es un componente importante, sobre todo cuando se analiza en un ambiente virtual donde esta se da de manera consciente y espontánea, pues cada sujeto decide si participa o no y en qué momento lo hace.

Como se aprecia en el gráfico, los sujetos afirman que la participación en este modelo instruccional conllevo a mejorar su proceso de redacción. De hecho, el rango de participación fue más frecuente y alto que en las clases presenciales.

Las aseveraciones anteriores suponen que el desarrollo de la competencia escrita en un ambiente colaborativo, donde se registran aportaciones y realimentación tanto del profesor como de los compañeros virtuales, guían la elaboración de escritos aceptables. Dichas aportaciones críticas se basan en aspectos fundamentales de los procesos de escritura tales como su propósito, audiencia, planificación y aspectos referentes a las teorías transaccionales (White y Bruning, 2005).
FLORES-GONZÁLEZ, Norma, FLORES-GONZÁLEZ, Efigenia, CASTELÁN-FLORES, Vianey y ZAMORAHERNANDEZ, Mónica. Objetos digitales de aprendizaje en entornos virtuales para fomentar la escritura: percepciones de los estudiantes. Revista de Tecnologías de la Información. 2020 
La corrección y edición de textos es más fácil y precisa con el uso de las herramientas digitales en plataforma Moodle

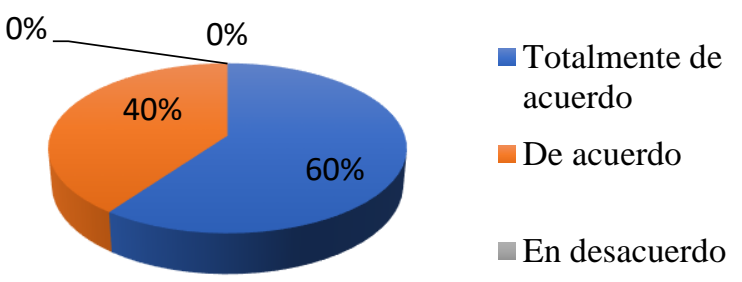

Gráfico 4 La corrección y edición de textos es más fácil y precisa con el uso de las herramientas digitales en plataforma Moodle

El proceso de revisión en la expresión escrita es muy complejo y requiere de un dominio de proficiencia en L2 aceptable por parte del escritor para mejorar la calidad comunicativa del texto.

En el caso del presente estudio, el $100 \%$ de los sujetos percibieron favorablemente el uso de herramientas digitales en plataforma Moodle como apoyo para la corrección y edición de sus textos. De hecho, es notorio que las herramientas digitales o software como Grammarly, Reverso, Language Tool, Whitesmoke Writer, Scribens, y Turnitin, componentes del diseño instruccional y de los ODA, fueron considerados como los más atractivos e innovadores al ser un medio viable para la revisión de textos.

Lo anterior coincide con Torres, Ayala \& Vinasco, (2010), quienes sostienen que los escritores pueden identificar y corregir sus errores al utilizar herramientas digitales $\mathrm{y}$ software, obteniendo un registro más formal, académico, y por tanto adecuado a su audiencia. Además, el uso de plataformas sofisticadas como Google-Docs permitió a los alumnos una interacción dinámica y sincrónica, la cual también abonó a la composición escrita.

Por otro lado, el uso de herramientas o software anti-plagio permitió a los autores de textos evitar copiar y pegar textos, dando prioridad a investigar, leer, interpretar, parafrasear y en general comprender antes de plasmar ideas.
La realimentación de mis compañeros de clase detona cambios en mi desempeño

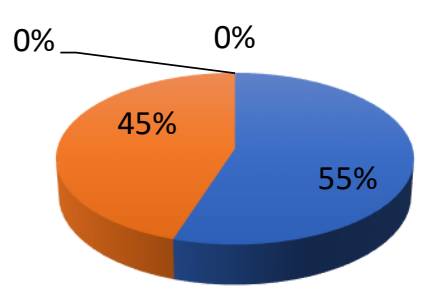

Totalmente de
acuerdo
De acuerdo
En desacuerdo
Totalmente en
desacuerdo

desacuerdo

Gráfico 5 La realimentación de mis compañeros de clase detona cambios en mi desempeño cuando redacto

Tomando en cuenta los resultados $(55 \%$ totalmente de acuerdo y $45 \%$ de acuerdo), es evidente que este modelo ofrece oportunidades para exponer a los estudiantes a un aprendizaje significativo con respecto a la escritura a través de la realimentación colaborativa por parte de sus lectores como son el profesor y compañeros virtuales (Hyland, 2002).

Los entornos virtuales y diseño de ODAS proveen temas importantes relacionados con mis estudios

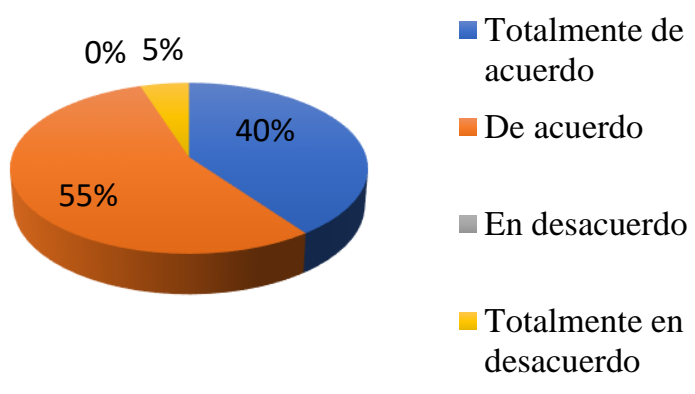

Gráfico 6 Los entornos virtuales y diseño de ODA proveen temas importantes relacionados con mis estudios

El 95\% de la muestra está de acuerdo en que el uso de la plataforma y diseño instruccional provee información relacionada con sus estudios. Esto debido a que en la primera fase que es la preparación para la redacción, los sujetos se ven expuestos a una seria de textos que proveen información como base para identificar y seleccionar contenido para elaborar un escrito. Dichos textos, fueron seleccionados de acuerdo con el mapa curricular de su carrera y a la enseñanza-aprendizaje del inglés como lengua extranjera. En general, la plataforma fue caracterizada como un espacio virtual con herramientas para comunicar, comprender, monitorear y evaluar el proceso de la competencia escrita.

FLORES-GONZÁLEZ, Norma, FLORES-GONZÁLEZ, Efigenia, CASTELÁN-FLORES, Vianey y ZAMORAHERNANDEZ, Mónica. Objetos digitales de aprendizaje en entornos virtuales para fomentar la escritura: percepciones de los estudiantes. Revista de Tecnologías de la Información. 2020 
Mi proceso de escritura mejora al utilizar estrategias de redacción con ayuda de las herramientas digitales

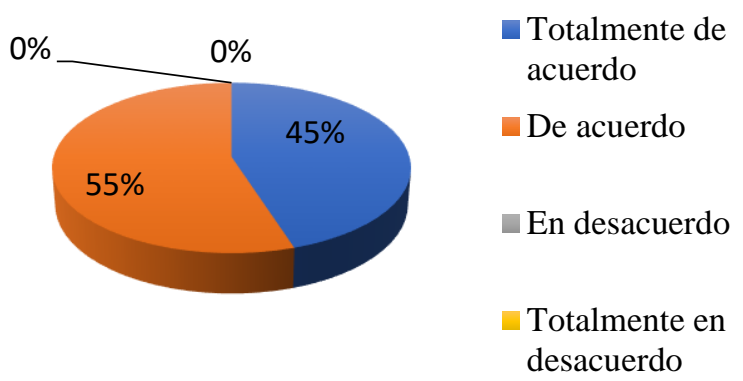

Gráfico $7 \mathrm{Mi}$ proceso de escritura mejora al utilizar estrategias de redacción con ayuda de las herramientas digitales

El gráfico muestra que el $55 \%$ está de acuerdo y el $45 \%$ totalmente de acuerdo, lo cual indica que hay una percepción altamente positiva con respecto al uso de estrategias de redacción apoyadas por herramientas digitales (Grammarly, Reverso, Language Tool, Scribens, Turnitin, etc.), para la redacción, revisión y edición del texto.

Además, a nivel macro-estructural, sus escritos mostraron relaciones de significado entre las secciones que lo constituyen, revelando una cohesión léxica.

También hay un avance positivo a nivel proposicional, donde se evidencia una mejora gradual de la estructuración morfosintáctica, sin errores de concordancia entre sustantivo y verbo, así como una relación semántica adecuada a la audiencia, al seleccionar el léxico apropiado. Por último, los textos muestran un dominio pleno de la ortografía.

Dichos resultados coinciden con Torres, Ayala \& Vinasco (2010) al señalar que el uso de herramientas beneficia el proceso de la competencia escrita.
El diseño instruccional fomenta la escritura efectiva, tomando en cuenta mis logros

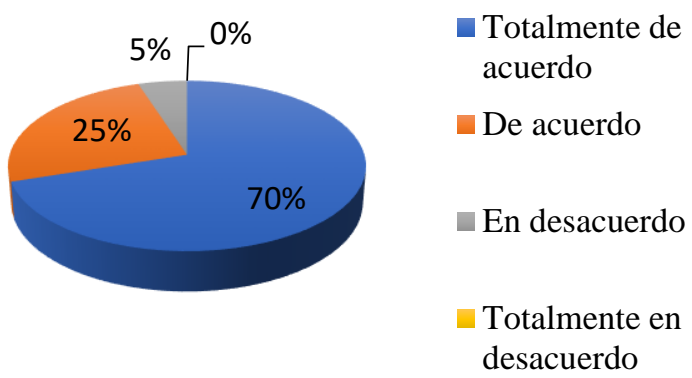

Gráfico $8 \mathrm{El}$ diseño instruccional fomenta la escritura efectiva, tomando en cuenta mis logros

El $70 \%$ de los sujetos manifiesta estar totalmente de acuerdo con la afirmación: el diseño instruccional fomenta la escritura efectiva, tomando en cuenta sus logros toda vez que dicho modelo contempla elementos como determinar el tema, definir propósitos, utilizar estrategias coherentes y precisas, entre otros.

Esto se corrobora con lo expuesto por González, Rodríguez y Ledo (2019) quienes indican que los elementos antes mencionados son básicos para diseñar y desarrollar herramientas computacionales que favorezcan la escritura en una Lengua Extrajera.

Por otra parte, el $25 \%$ asegura estar de acuerdo. Esto concuerda con Barritt, Chuck \& Alderman (2004), ya que el diseño de los objetos digitales de aprendizaje empleados para desarrollar la escritura, estuvieron basados en experiencias reales y ricas en significado, lo que motivo a los estudiantes al satisfacer sus necesidades tecnológicas y de escritura.

Es importante mencionar que el $5 \%$ de la muestra estuvo en desacuerdo. Este es un caso aislado y viable para analizar, pues existen distintos factores que pudieron detonar dicha afirmación, por ejemplo, el acceso a la red, la disposición y el espacio.
FLORES-GONZÁLEZ, Norma, FLORES-GONZÁLEZ, Efigenia, CASTELÁN-FLORES, Vianey y ZAMORAHERNANDEZ, Mónica. Objetos digitales de aprendizaje en entornos virtuales para fomentar la escritura: percepciones de los estudiantes. Revista de Tecnologías de la Información. 2020 


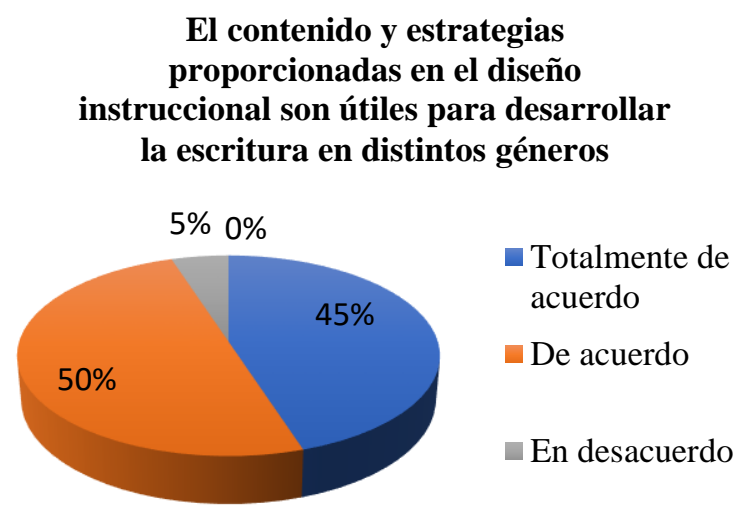

Gráfico 9 El contenido y estrategias proporcionadas en el diseño instruccional son útiles para desarrollar la escritura en distintos géneros

La información y las estrategias proporcionadas en el diseño instruccional fueron percibidos como una herramienta para desarrollar un aprendizaje significativo en el ámbito de la escritura.

El 95\% de la población objetivo expresa una satisfacción positiva. Esto se corrobora con los estudios de Haan y van Esch (2005), quienes demuestran que la práctica de la habilidad escrita a través de un entorno virtual favorece la fluidez como detonante para escribir oraciones complejas, elaboradas, y escritos extensos.

Los resultados también se correlacionan con los estudios de Greene (2000) e Hirvela (2005), toda vez que las estrategias y los textos empleados demandaron un pensamiento crítico para el proceso de composición y redacción en distintos géneros. Dichos aspectos fueron reconocidos y valorados por los estudiantes al ser útiles para el desarrollo de su competencia escrita.

Recomiendo este modelo de aprendizaje a partir de mi experiencia y resultados

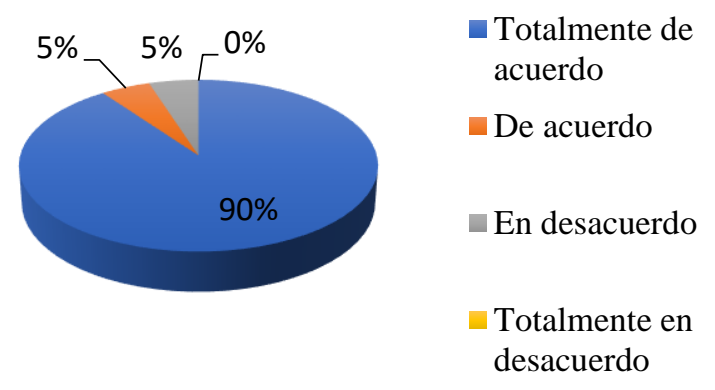

Gráfico 10 Recomiendo este modelo de aprendizaje a partir de mi experiencia y resultados
Como se observa, el 5\% de los sujetos está de acuerdo y el $90 \%$ totalmente de acuerdo, lo cual nuevamente indica que casi toda la población ve como plausible el modelo para fomentar la escritura.

La apreciación de los sujetos desde su posición de usuarios de la plataforma y diseño instruccional indica que el uso de entornos virtuales y herramientas digitales son atractivos y útiles para los estudiantes de nivel superior al ofrecer no solo el desarrollo de la escritura académica sino también el aprendizaje de la L2 en la comprensión lectora y enriquecimiento léxico. Dicha caracterización dio pauta a la recomendación del modelo de aprendizaje.

En general, el modelo influyó positivamente en la producción escrita de los alumnos en términos de calidad y proficiencia del idioma.

\section{Conclusiones}

Los sujetos reconocieron a los ODA implementados en la plataforma virtual como un medio idóneo para interactuar lingüísticamente y organizar sistemáticamente sus pensamientos en textos escritos.

En consonancia con lo anterior, cabe mencionar que la percepción de los sujetos con respecto al diseño instruccional para fomentar la escritura se caracteriza por:

- $\quad$ El uso de diferentes patrones tomando en cuenta el tema y la audiencia.

- El trabajo colaborativo, particularmente en el proceso de revisión, el cual permite una realimentación bidireccional, conduciendo a experiencias significativas para el desarrollo de la escritura funcional en el idioma inglés.

- La motivación y confianza que brindan los ODA para escribir textos de distintos géneros así como el incremento de tareas y actividades investigativas mediante la búsqueda, selección, organización y sistematización de la información con apoyo de herramientas digitales. 
- La elección de herramientas digitales y estrategias de redacción que permitieron escritos sin errores gramaticales, ortográficos y de puntuación, generando a su vez, aprendizaje con respecto a su uso en contextos reales.

La identificación de herramientas digitales $\mathrm{y}$ actividades que activan el uso de estrategias para desarrollar dicha competencia

Los alumnos también percibieron el modelo instruccional como un escenario de interacción factible y proactivo con respecto a la participación, lugar donde convergen la reflexión, el pensamiento crítico-reflexivo y sobre todo el análisis en el texto discursivo.

Lo anterior, constituye una aportación a las prácticas de enseñanza de la competencia escrita como una posible alternativa para su desarrollo de manera activa, innovadora y atractiva.

En resumen, se conocieron las percepciones que los sujetos tienen sobre los ODA y cómo el material digital permitió la generación del conocimiento, la mejora de sus competencias linguiísticas y realización de actividades escritas con elementos de cohesión sólidos.

\section{Referencias}

Barritt, Chuck \& Lee Alderman. (2004). Creating a reusable learning objects strategy: Leveraging Information and Learning in a Knowledge Economy. San Francisco: Pfeiffer Publisher.

Bazerman, C., Little, J., Bethel, L., Chavkin, T., Fouquette, D., \& Garufis, J. (2005). Reference Guide to Writing Across the Curriculum. West Lafayette: Parolor Press.

Beltrán Llera, J. (2003) "Enseñar a aprender". Enhttp://www.ucm.es/info/psicevol/CURRICU LUMS/ENSENAR\%20A\%20APRENDER.htm . Consultado el 26 de Noviembre del 2020.

Berrios, M. (2004). Las Tecnologías de la Información y la Comunicación (TIC) y Los Adolescentes. Algunos Datos. Universidad de Barcelona, España.
Burgos, J. V. (2009). Innovación e investigación con recursos educativos abiertos (REA): Casos prácticos para el ámbito educativo. Disponible en Escuelas de Graduados en educación de la universidad virtual del tecnológico de Monterrey. Recuperado el 25 de noviembre del 2020 en: http://sesionvod.itesm.mx/acmcontent/9ecfbd19 -32da-4816-b003-

83c1523b17a5/UnspecifiedEGE

Canale, M. \& Swain, M. (1980). Theoretical bases of communicative approaches to second language teaching and testing. Londres, Inglaterra:Longman.

Cassany, D. (2005). La expresión escrita en el aula de E/LE. Madrid: Arcolibros.

Chao, Y. J., Lo, H. (2009). Students' perceptions of Wiki-based collaborative writing for learners of English as a foreign language, Interactive Learning Environments, First published on: 15 October 2009 (iFirst).

Enríquez, S. C. (2011). La enseñanza de la escritura en entornos virtuales. Puertas Abiertas, (7).

Flores-González, E., Flores-González, N., Fernández-Crispín, A. (2018). Aplicación de la estrategia mapas conceptuales para el desarrollo de la competencia comprensión lectora en Biología. Revista Teoría Educativa. 2 (3), 2029.

García, A. (2007). La expresión escrita de los alumnos de etnia gitana, escolarizada en educación primaria. Redalyc. 3(1), 437-448.

Garrison, D y Anderson, T. (2005). El e-learning en el siglo XXI: Investigación práctica. Barcelona: Octaedro.

Gisbert, M., Cabero, J. y Llorente, M. (2007). El papel del profesor y el estudiante en los entornos tecnológicos de formación. En J. Cabero (Coord.) Tecnología educativa (p.p. 253-280). Madrid: Mc Graw Hill.
FLORES-GONZÁLEZ, Norma, FLORES-GONZÁLEZ, Efigenia, CASTELÁN-FLORES, Vianey y ZAMORAHERNANDEZ, Mónica. Objetos digitales de aprendizaje en entornos virtuales para fomentar la escritura: percepciones de los estudiantes. Revista de Tecnologías de la Información. 2020 
González, J. García-Romeu, J. (2010). Aprender escribiendo un wiki para el desarrollo de las estrategias de expresión escrita de forma cooperativa a través del proceso de composición- I Jornadas Internacionales sobre el uso de las tecnologías de la información y la comunicación en la enseñanza del español como lengua extranjera, Madrid, 28-30 de junio 2010

http://www.educacion.gob.es/redele/jornadasint .shtml

González, A., Rodríguez, M., Ledo, M. (2019). Enseñar a escribir en inglés: el enfoque procesoproducto orientado a la acción. Transformación. 15 (1), 14-26.

Greene, D. (2000). A design model for beginnerlevel computer-mediated EFL writing. Computer-Assisted Language Learning, 13 (3), 239-259.

Haan, P. \& van Esch, K. (2005). The development of writing in English and Spanish as foreign lenguages. Assessing Writing, 10 (2), 100-116.

Hirvela, A. (2005). Computer-based reading and writing across the curriculum: Two case studies of L2 Writers. Computers and Composotion, 22 (3), 337-356.

Hyland, K. (2002). Teaching and Researching: Writing. Londres, Inglaterra: Longman.

Kessler, G. (2009). Student-initiated Attention to Form in Wiki-based Collaborative Writing in Language Learning \& Technology, 13(1), 79-95.

López, A., Romero, S. I. y Ramírez, M. S. (2008). Utilización de objetos de aprendizaje como opción para la educación continua de los docentes de nivel superior. Memorias del primer congreso nacional de ciencias humanas: Gestión de competencias en la sociedad de conocimiento. Pachuca, Hidalgo.

Paquienséguy, F., \& Pérez Frangoso, C. (EneroJunio de 2010). El aprendizaje en línea: Una forma de atender las necesidades de poblaciones estudiantiles diversas. Revista Q, 4 (8), 1-10. Obtenidos de http://revistaq.upd.edu.co
Peyton, Joy K. et al. (1994). Implementing Writing Workshop with ESOL students: Visions and realities. En TESOL Quarterly, 28 (3).

Prior, P. (2006). A sociocultural theory of writing. En C. MacArthur, S. Graham \& J. Fitzgerald (Eds.), Handbook of writing research (pp. 54-66). New York: The Guilford Press.

Quiroz, J. S. (2011). Diseño y moderación de entornos virtuales de aprendizaje (EVA) Editorial UOC.

Salvador, F. (2000). El análisis cualitativo: un ejemplo de empleo de MCC (método comparativo constante). Primera parte. En infancia en Red. Proyecto Margarita.

Serrano, S. y Peña, J. (2003). La escritura en el medio escolar: Un estudio en las etapas. Educare, 6(20), 397-407. Recuperado de http://www.saber.ula.ve/bitstream/123456789/1 9747/1/articulo6.pdf

Sharma, P., \& Barrett, B. (2007). Blended learning: Using technology in and beyond the language classroom. Oxford: Macmillan.

Torres, M. I. B., Ayala, O. E. H., \& Vinasco, V. V. (2010). El desarrollo de la escritura académica en el ambiente virtual Lingweb: realidades y desafíos. Lenguaje, 38 (2), 351-386.

Ward, J. (2004). Blog Assisted Language Learning: Push Button Publishing for the Pupils. TEFL Web Journal, 3 (1), 1-15.

White, M. J., \& Bruning, R. (2005). Implicit Writing Beliefs and their Relation to Writing Quality. Contemporary Educational Psychology, 30, 166-189. doi: 10.1016/j.cedpsych.2004.07.002

Willey, D. (2000). Connecting learning objects to instructional design theory: a definition, a metaphor, and a taxonomy. The instructional Use of.

Wu, W. (2005). Using Blogs in an EFL Writing Class. 2005 International Conference on TEFL and Applied Linguistics www.chu.edu.tw/wswu/publications/papers/con ferences/05.pdf 
Zhang, D. (2009). The Application of Blog in English Writing. Journal of Cambridge Studies, $4(1)$ 